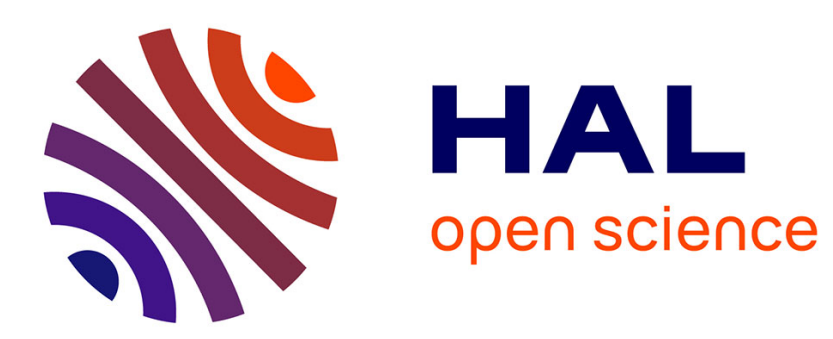

\title{
Integrated approach based on high level Petri Nets for evaluating Networked Control Systems
}

\author{
Belynda Brahimi, Eric Rondeau, Christophe Aubrun
}

\section{To cite this version:}

Belynda Brahimi, Eric Rondeau, Christophe Aubrun. Integrated approach based on high level Petri Nets for evaluating Networked Control Systems. 16th Mediterranean Conference on Control and Automation, MED'08, Jun 2008, Ajaccio, France. pp.1118-1123, 10.1109/MED.2008.4602274 . hal00347434

\section{HAL Id: hal-00347434 \\ https://hal.science/hal-00347434}

Submitted on 16 Dec 2008

HAL is a multi-disciplinary open access archive for the deposit and dissemination of scientific research documents, whether they are published or not. The documents may come from teaching and research institutions in France or abroad, or from public or private research centers.
L'archive ouverte pluridisciplinaire HAL, est destinée au dépôt et à la diffusion de documents scientifiques de niveau recherche, publiés ou non, émanant des établissements d'enseignement et de recherche français ou étrangers, des laboratoires publics ou privés. 


\title{
Integrated Approach based on High Level Petri Nets for Evaluating Networked Control Systems
}

\author{
B. Brahimi, E. Rondeau and C. Aubrun
}

\begin{abstract}
The aim of this paper is to propose an integrated modelling environment to represent globally the Networked Control Systems (NCS) behaviour by using the High Level Petri Nets (HLPN) formalism. This work considers NCS based on switched Ethernet architectures. This communication architecture is modelled with HLPN and is evaluated according to different scheduling mechanisms and traffic load. After that, NCS model based on HLPN is proposed. This model integrates the Ethernet switch model and applicative environment of NCS: controller, process... Finally, a strategy to control the network in order to adapt the Quality of Service $(\mathrm{QoS})$ according to the quality of Control (QoC) required by the application is proposed. This strategy is based on a Weighted Round Robin policy. The obtained results show that the scheduling mechanisms enable to improve the performance of communication system and then to improve the application performances.
\end{abstract}

\section{INTRODUCTION}

$\mathrm{R}$ ecent technological advances have enabled distributed control systems to be implemented via network. This allows feedback control systems to be implemented on the shared network. These systems are called NCS (Networked Control Systems). This kind of implementation has several advantages, such as: reduced wiring costs, easier system diagnosis and maintenance, and improved modularity and flexibility in the system design [1]. However, one issue inherent to NCS is the induced network delay that occurs while exchanging data between the devices connected to the shared network. This delay can be constant or time varying, important or infinite (loss packet case). Then, new methods to analyse and design the NCS are required and the pluridisciplinary aspect of NCS imposes deep knowledge in automatic control theory, computer and communication networks. The NCS finds its bases since the 1980's in the research community working on Industrial Loal Area Network and on fieldbuses. Ray and Wittenmark contributed in research on timing problems in real-time control systems.

B. Brahimi is with the Automatic research center of Nancy (CRAN) faculté des sciences BP 239. 54506 Vandoeuvre Les Nancy, FRANCE. (phone: 333-8368-4441; fax: 333-8368-4459: belynda.brahimi@ cran.uhpnancy.fr).

E. Rondeau, is with the Automatic research center of Nancy (CRAN) faculté des sciences BP 239. 54506 Vandoeuvre Les Nancy, FRANCE. (phone: 333-8368-4425; fax: 333-8368-4459: eric.rondeau@ cran.uhpnancy.fr).

C. Aubrun is with the Automatic research center of Nancy (CRAN) faculté des sciences BP 239. 54506 Vandoeuvre Les Nancy, FRANCE. (phone: 333-8368-4463; fax: 333-8368-4459: christophe.aubrun@, cran.uhp-nancy.fr).
Since 2000 several working groups (Automatic and Telecommunication Networks, control systems and communication network) have emerged. The NCS was then called [19] (ICCS: Integrated Communication and Control Systems). The goal of these working groups is to unify the vocabulary of researcher from various domain working in the fieldNCS. In 2004, the European commission supports NeCST project (Networked control systems tolerant to fault). In 2005, at the 16th IFAC world congress in Prague, one technical committee (T.C-1.5) was created on the Networked Systems. Until now, this topic continues to present a great interest for industrial and research communities. For example, the European commission proposed a dedicated action on "Networked Embedded \& Control Systems" in 2007.

The main goal of the NCS is to control and/or to adapt the control law (quality of control: QoC) or the communication system (quality of service: QoS) to guarantee the overall systems stability. Two approaches are investigated:

- In the first approach, the application is adapted regarding to the network performances. This approach is mainly studied by the control researchers and consists in designing the control strategies by taking into account the delay and/ or packet loss induced by the network in the feedback control systems. This approach is referred as 'control over network'.

- In the second approach, the network is adapted (control of network) to provide a satisfactory level of QoS for the application. This approach is mainly studied by the network researchers and consists in designing real-time protocol communications and predictable timing behaviour protocols $[3,4]$.

Finally, these two complementary approaches can be combined by considering simultaneously QoC and QoS in order to adapt in a coordinated way, the control of process and the control of network. This combined approach is called co-design and requires new simulation tools for assessing NCS. The simulation tools have to be able to represent the behaviour of controllers, process, and networks.

This paper presents such tool based on Petri Nets modelling. The first section starts with a brief review of previous work on NCS. The second section emphasizes on the network modelling. A switched Ethernet network is considered in this study because as it now currently used in industrial application. The third section presents an approach to estimate the QoC finally used for optimizing the QoS offered by the network and also for evaluating the global NCS performances. 


\section{StATE Of ART}

\section{A. Introduction}

Three main approaches [17] to design and study the NCS are Classified as follows: The first approach is mainly studied by the control researchers and deals with control strategies taking into a count the delay and/or packet loss induced by network (control over network). The second approach is mainly studied by the network researchers and deals with control and/or adaptation methods (control of network) of network protocols regarding to the quality of Control required by the application. The third approach merges the two first ones.

\section{B. First Approach}

Nilsson [18] analyzes NCS in the discrete-time domain, and he models the network delay as constant delays, independent random delays and random delays represented under Markov chains. And he solves the LQG optimal control problem from the different delay models.

Goktas [5] designs a networked controller in the frequency domain using robust control theory. This method is based on robust control. The main advantage of this method relies in the fact that it does not require a priori information about the probability distributions of network delays. In the robust control theory, the network delays $\tau_{\text {ca }}$ (delay between the controller and actuator) and $\tau_{\mathrm{sc}}$ (delay between sensor and controller) are modelled as simultaneous multiplicative perturbation. Both delays are assumed to be bounded and able to be approximated by the fluid-flow model [6] or network calculus theory [7].

\section{Second Approach}

In [8] analytic proofs of stability for networked control systems are given for common statistically protocols, and a new protocol: Try-Once-Discard (TOD) enabling to provide quality of service required by the application. This protocol is based on the dynamic scheduling, in which the node with the greatest weighted error from its last reported value (to the controller) transmits its message. Again, the Maximum Allowable Transfer Interval (MATI) constraint ensures at least one such transmission every $\tau$ seconds. However, TOD does not guarantee that each node will transmit once every $p$ transmissions. Others researchers improve the existing protocols [3, 9] or add protocol layer over existing protocols to improve or guarantee the temporal performances of the network. Hoang et al. [10] develop a technique that supports a mix of real-time (RT) and non-real-time (standard IP) traffic coexisting in a switch-based Ethernet network. The RT traffic is scheduled according to the Earliest Deadline First policy and its timeliness is guaranteed by means of adequate on-line admission control. The proposed system architecture requires the addition of a real-time layer (RT-1) on network components, either end nodes or the switch. The RT-1 is responsible for establishing real-time connections, performing admission control, providing time synchronization, and finally managing the message transmission and reception of both real-time and non-real- time traffic classes. [11] introduce the traffic smoothing technique. The authors show in a analytical way that it is possible to provide a probabilistic guarantee that packets may be successfully transmitted within a pre-defined time bound, if the total arrival rate of new packets generated by all the stations on the network is kept below a threshold, called network-wide input limit. An interface layer called traffic smoother is located over the Ethernet data link layer. This element shapes the traffic generated by the respective node according to a desired rate commonly referred as station input limit. On one hand, the traffic smoother implements a leaky bucket mechanism with appropriate depth and rate, that captures and smoothes the non-real-time traffic generated by the node. On the other hand, the realtime traffic is non-bursty thus spaced in time, with short payloads, resulting in low collision probability. Thus, it does not need smoothing filter and it is immediately sent to the network, thru the leaky bucket. Lo Bello et al. [12] develop a further evolution of the dynamic approach that consists in estimating the network load on-line using two parameters: the number of collisions and the throughput, both observed in a given interval. These parameters are then fed to a fuzzy controller to set the instantaneous station input limit. The resulting efficiency is substantially higher compared with both the static and the dynamic approaches.

\section{Third Approach}

[13] consider the scheduling network problem and the optimal control of systems simultaneously. The deterministic network is chosen. The proposed method is based on the quadratic cost function used to evaluate the control performances and adaptive scheduling to improve the performance of system. The network is assumed bandwitdh bounded i.e. in each sampling interval only $\mathrm{b}$ control messages can be transmit with $\mathrm{b} \leq \mathrm{m}$ and $\mathrm{m}$ is total control messages to transmit. [14] consider a set of NCS which are connected via a network. Each plant transmits sensors data at transmission period $h \mathrm{i}(\mathrm{t})$. One can compute the transmission period bounds which guarantee stability for each individual plant. However, when the transmission path is shared with other NCS, transmission scheduling among the plants has to be performed. A set of NCS transmissions is said to be schedulable by a scheduling algorithm (or the transmission schedule) is feasible if all transmissions can be completed before deadlines are reached. The authors use the Rate Monotonic (RM) scheduling algorithm, where tasks with shorter periods have higher priorities. RM is a fixed-priority assignment: priorities are assigned to tasks before execution and do not change over time. Moreover, RM can be preemptive: the currently executing task is preempted by a newly-arrived task with shorter period.

\section{E. Conclusion}

The objective of this work is to propose a unified environment for modelling NCS. The goal is to be able to simulate the behaviours of a process, control system, and network with the same tool. Also, we propose a method based on control of scheduling mechanism in network to 
reduce network induced delays. We choose High Level Petri Nets (HLPN) formalism to model the NCS. In this paper, the network interconnecting the distributed system is a switched Ethernet which is modelled in the next section. The figure 1 illustrates the method used in this paper to model, analyse and verify the NCS performance.

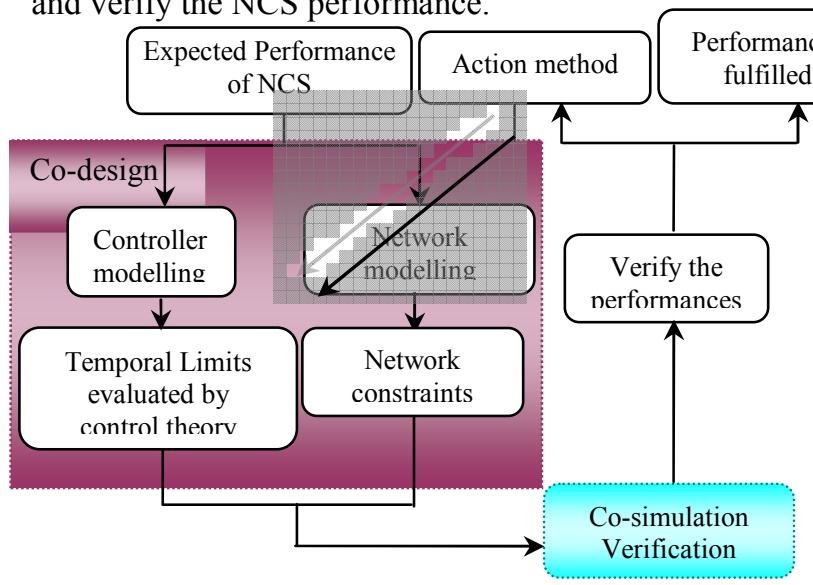

Fig. 1. Synoptic of integrated method proposed

\section{Switched ETHERNET ModELING}

\section{A. Introduction}

HTCPN is used for modelling communications because PN is a formal method enabling to express parallelism, synchronization, interaction, resource sharing, temporal (and stochastic) properties, and to achieve qualitative analysis (checking of the logic of the non-temporal and/or temporal mechanisms) and quantitative analysis (performance evaluation and/or reliability). Then, this formalism offers a framework well adapted and progressive for the representation and the analysis of the communication systems. This model uses a generic and modular approach which requires the use of 'Colouring' and Hierarchy. Others authors used this formalism to model the Ethernet switch [15].

\section{B. Ethernet Switch Architecture}

The received frames are stored in the global shared memory (8MB) to avoid Head of Line Blocking (HOL) problem.
This switch moves from input port to destination port by using switching fabric based on switching matrix (3.2 Gbps). Before the transmission to the destination, the frames are stored and classified in the output buffers regarding its destination and priority level. The frames are, after that, scheduled by the scheduling algorithms before transmission (figure 2). Two scheduling algorithms are implemented in this switch: the Priority Strict algorithm (PS) and the Weighted Round Robin algorithms (WRR). The switch is modelled from a hierarchy of models.

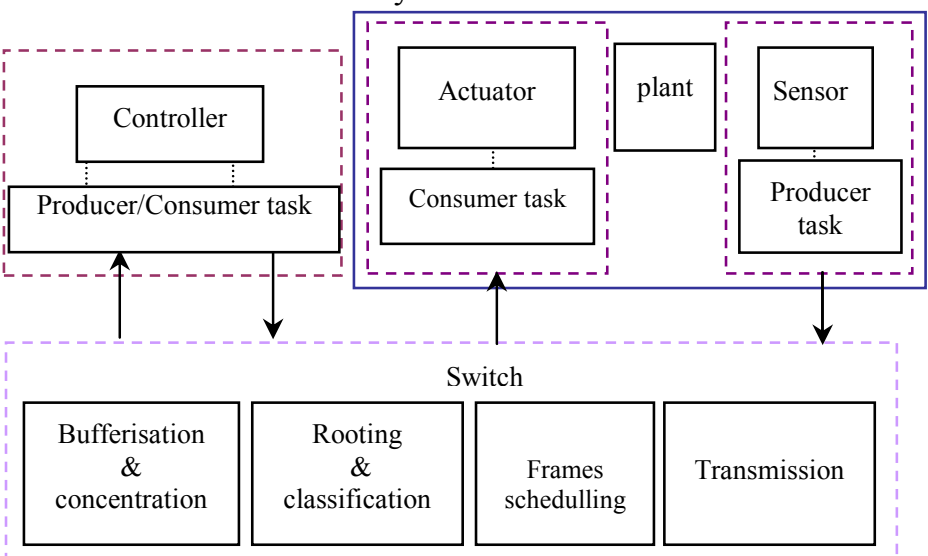

Fig. 2. Modelled system: The switch in periodic producer/consumer relationship.

The operations include in the switch are modelled by using HTCPN formalism in periodic producer/consumer relationship (figure 2). This relationship represents the controller-actuator and sensor-controller traffic behaviour [16]. Only some parts are briefly presented in this paper (figure 3):

- Producer: The frames are modelled by the tokens in the Petri nets models. The colour of the tokens models the different attributes of the Ethernet frame (destination and source addresses, the data, and the priority level). Three levels of priorities are used. Obviously, other attributes can be added. Each triplet supports temporal stamp. The producer is illustrated by traffic source (figure 3 ). The frames are generated periodically (period d). This period is illustrates by the stamp d associated to the transition Ts1.

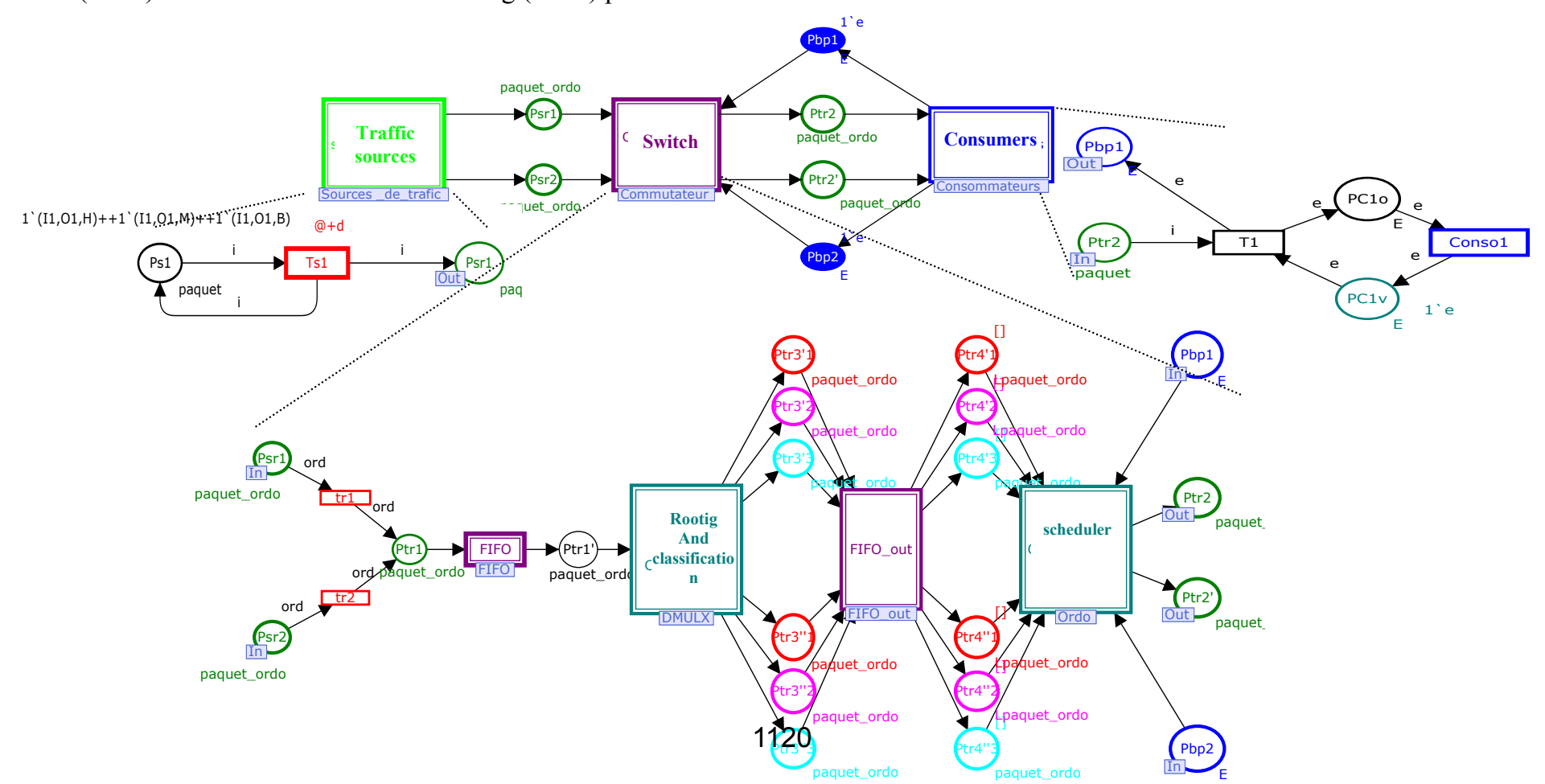

Fig. 3. HTCPN model of switch: only producer, consumer and one level of switch model hierarchy is illustrated. The whole model is presented in [2] 
This period enables to model the sampling period of sensors and actuators.

- Switch: The frames generated by the traffic source, are buffered in the shared memory presented by the substitution transition $^{1}$ (FIFO). After that, they are rooted and classified according to destination and priority level associated (rooting and classification substitution transition). There are as many queues as of priorities. Next, the frames are buffered in the output buffers (FIFO_Out substitution transition). Finally, they are scheduled, regarding two scheduling algorithms: Either SP or WRR (scheduler substitution transition).

\section{Simulation results}

The switch Ethernet model is simulated by using CPNTools software, according to the load and the scheduling algorithm variation. The goal of the simulation is to show the impact of scheduling methods on the traffic and then the interest to manage the scheduling methods in the context of NCS. In this simulation, three messages are periodically sent (period $10 \mathrm{~ms}$ ) to the Ethernet switch. A priority is associated to each message, respectively: High priority (HP), Mean (MP) or Low (LP). The behaviour of (HP) traffic is only analysed, it could represent the real-time communications between the controller and the process. The figure 4 shows the end-toend delay of high priority frames (HP) of Switch model simulated without scheduler, with PS and WRR schedulers. In the WRR scheduler, two weights are considered: when $10 \%$ of bandwidth is offered to the HP traffic and when $99 \%$ of bandwidth is offered to the HP traffic.

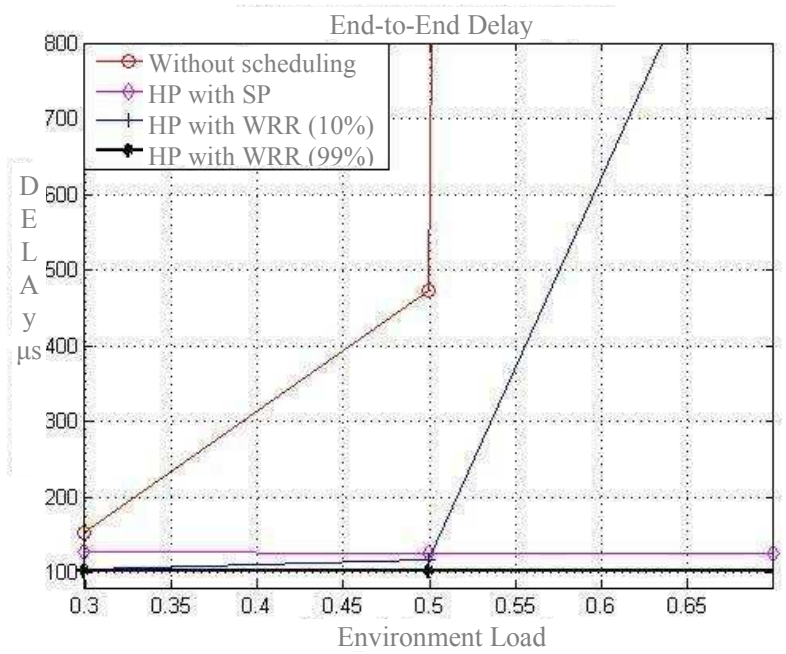

Fig. 4. Simulation results of CPN model of Ethernet switch according the scheduling policies and load environment

The results (figure 4) show that the behaviour of switch with WRR scheduler tuned to $99 \%$ is closed to the switch with PS scheduler behaviour. The interest of this simulation is to observe when no scheduler is implemented the real-time messages could be affected by other traffic. The same situation is observed, when the level of bandwidth offered to the real-time messages is not enough for WRR. In NCS

\footnotetext{
1 The substitution transition represents an abstraction of one part of coloured Petri net model. This part is detailed in apart.
}

context, the conclusion of the simulation is: it is mandatory to have mechanisms such as scheduler for protecting the real-time communications against others traffics using the same transmission support. Thus, the general matter in NCS is to find an optimised solution both offering enough bandwidth for non real-time traffic (to avoid famine for non real-time traffic) and ensuring satisfactory bandwidth for real-time traffic regarding to the constraints defined by the QoC.

\section{NeTWORKED CONTROL SySTEM MODELING}

\section{A. Performance Index Evaluation}

In this section, the delay tolerated by the feedback control system is calculated by an iterative procedure based on the necessary conditions for system stability. The discrete time system is represented by (1).

$$
x(k+1)=A x(k)+B u(k)
$$

Where $\mathrm{k}$ is the time index, and $x \in \mathfrak{R}^{n}$ et $u \in \mathfrak{R}^{l}$ are the state and input vectors, respectively. $A \in \mathfrak{R}^{n \times n}$ et $B \in \mathfrak{R}^{n \times l}$ are coefficient matrices obtained from the continuous-time model.

The conditions for asymptotic stability making a controlled process stay in its allowed state space can be used to derive this threshold called making decision threshold (MDT). Two cases are considered:

- Delay $<T s$ (Ts is the sampling period): The equation is as following,

$$
x(k+1)=A x(k)+B_{1} u(k)+B_{2} u(k-1)
$$

Where:

$$
B_{1}=\int_{0}^{T_{s}-\Delta} e^{A c(S)} d s B_{c} \quad B_{2}=\int_{T_{s}-\Delta}^{T_{s}} e^{A c(S)} d s B_{c}
$$

- Delay $>T s$ :

The delay (MDT) is assumed to be stochastically stationary, which corresponds to the characteristics of network induced delay (the frame can be transmitted or not). This property can be represented by a binomially distributed random variable. The system dynamics are modified according to the assumed maximum delay, NTs, and the probability distribution of delays whose occurrence periods $\leq \mathrm{N} T s$, where $\mathrm{N}$ is changed from 1 to the actual maximum delay (or MDT) denoted by DTs. The pole positions of the modified state equation will then be tested to derive necessary conditions for (asymptotic) system stability. To estimate the delay (MDT) the following augmented state results:

$$
\begin{gathered}
X(m+1)=A_{D} X(m)+B_{D i}^{1} U(m)+B_{D i}^{2} U(m+1) \\
U(m)=-F_{D} X(m)
\end{gathered}
$$

The equations $[4,5]$ are obtained by assuming that the control inputs were not updated for $i$ sampling periods from that due to a delay where $0 \leq i \leq N$ (i.e. the delay is equal to $i$ sampling periods).

By combining the delay distribution and equation 4, the following equation, results:

$$
X(m+1)=A_{D} X(m)+\sum_{i=0}^{N} \xi_{i}\left(B_{D i}^{1} U(m)+B_{D i}^{2} U(m+1)\right)
$$


Where $\xi_{i} \in\{0,1\} \quad \xi_{i} \in\{0,1\}$ is a binomially-distributed random variable with parameter $q_{i}$, i.e. $\operatorname{Pr}\left[\xi_{i}=1\right]=q_{i}$. Then we calculate characteristic equation of system:

$$
\operatorname{det}\left[\left(I+\sum_{i=0}^{N} q_{i} B_{D i}^{2} F_{D}\right) z^{N}-A_{D}+\sum_{i=0}^{N} q_{i} B_{D i}^{1} F_{D}\right]=0
$$

The asymptotic stability can be tested with the pole positions of (8). The characteristic equation of the zero-delay case (i.e.

$$
\begin{aligned}
\left.q_{0}=1 \text { et } P=\sum_{=1}^{N} q_{i}=0\right) \text { is: } & \\
& \operatorname{det}\left[z^{N} I-(A-B F)^{N}\right]=0
\end{aligned}
$$

Further, one can get the following characteristic equation for the worst case in which $q_{N}=1$. The control input is updated only once every $\mathrm{N}$ sampling intervals due to the periodic delay of an active duration NTs:

$$
\operatorname{det}\left[z^{N} I-A^{N}+\sum_{i=0}^{N-1} A^{i} B F\right]=0
$$

Where, $A=Q D Q^{1}$ by similarity transformation if $D=\operatorname{diag}\left[d_{1}, \ldots, d_{n}\right]$ and $d_{i}$ are the eigenvalues of A.

\section{B. Integrated Approach Evaluation}

In this section, the NCS is modelled by using HTCPN. The Ethernet switch model is extended by modelling controller, actuator, process and sensor. The method to reduce the network induced delay is also modelled. The principle is: if the frames delay, is upper than the evaluated MDT then we have to act on the scheduling mechanisms to reduce the delay. In this work the acting method consists in increasing the bandwidth for the high priority frames (figure 5). The high priority frames represent the control signals.

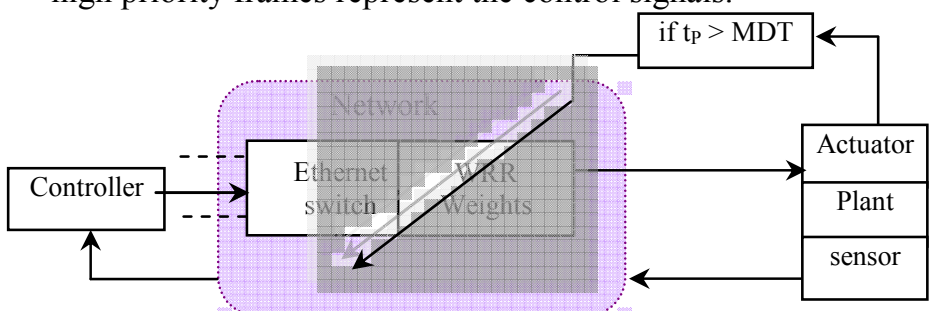

Fig 5. Integrated modelling and strategy of scheduling mechanisms control

\section{Example}

Consider the simple controlled process:

$$
x(k+1)=1.05 x(k)+1.8 u(k)
$$

Where $Q=2, R=7$, the optimal feedback control is $F=0.3594 . T e=1 \mathrm{~ms}$. The figure 6 represents the CPN model of the simple controlled process. The Equation 10 is associated to 'plant' transition that when it is fired the next state of the system is computed. The same approach (not presented in this paper) enables to model the controller.

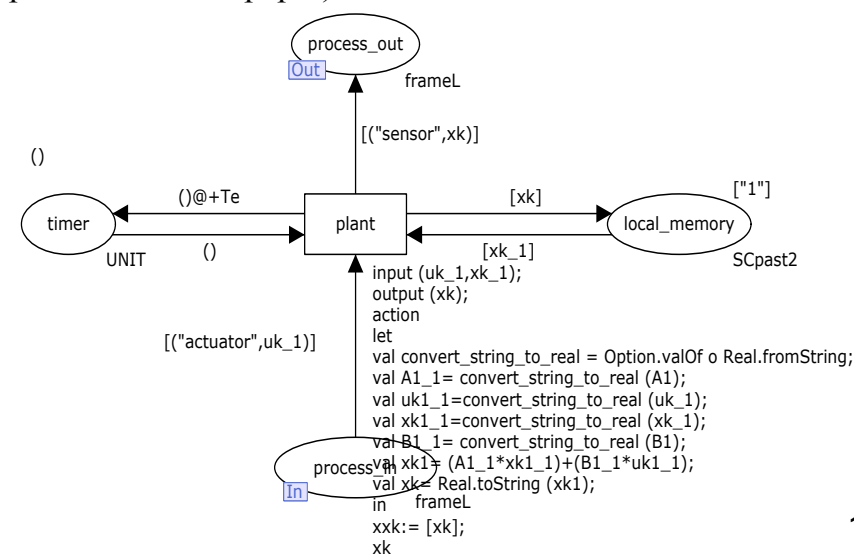

\section{QoC: Performance index evaluation}

The threshold delay to make decision to act on the scheduling mechanisms of network is computed by the iterative test of pole positions of the system, for different $\mathrm{N}$ in the case where $q_{N}=1$, and MDT $=3$ Te.

\begin{tabular}{|c|c|c|}
\hline$N$ & Equation & $|\lambda|$ \\
\hline 2 & $E i g\left(A^{2}-A B F-B F\right)$ & 0.2237 \\
\hline 3 & $E i g\left(A^{3}-A^{2} B F-A B F-B F\right)$ & 0.8818 \\
\hline 4 & $\operatorname{Eig}\left(A^{4}-A^{3} B F-A^{2} B F-A B F-B F\right)$ & 1.5728 \\
\hline
\end{tabular}

Table 1. Relationship between pole positions and $N$ when $P=q_{N}=1$

\section{QoS assumptions}

The NCS is shared on the switch Ethernet with a bandwidth of $10 \mathrm{Mbit} / \mathrm{s}$. The real time traffic (RT) (frames that contain control signals) is periodic $(\mathrm{Te}=1 \mathrm{~ms})$ the length of the frames chosen is 64 octets $(51,2 \mu \mathrm{s})$ which correspond to the minimal size of an Ethernet frame. The non-real time traffic (NRT) (additional traffic) is periodic $(\mathrm{P}=1 \mathrm{~ms})$, the length chosen is 64 octets and 13 sources are implemented. The priority level (HP: high priority, MP: mean priority, LP: Low priority) and WRR scheduler are implemented. Three weights are modelled in the WRR: wlcorresponds to the bandwidth allowed for RT traffic (HP traffic), w2 and $w 3$ correspond to the bandwidth allowed for NRT traffic (MP and LP traffic). Two Weight configurations are considered: C1 $-w 1=10 \%, w 2=45 \%$ and $w 3=45 \%, \mathbf{C 2}-w 1=99 \%$, $w 2=0.009 \%$ and $w 3=0.001 \%$.

\section{QoS/QoC adaptation}

The NCS model based on HTCPN represented in figure 5 is simulated with CPNTools. The results show an important overshot (figure 7 a) due to the network induced delay $(5,1744 \mathrm{~ms})$. This delay (figure $7 . \mathrm{b})$ is upper than MDT= $3 \mathrm{~ms}$, then it causes the changing the weight of the WRR by switching from $\mathrm{C} 1$ (initial configuration) to $\mathrm{C} 2$. This is illustrated by the switching from 0 to 1 (figure $7 \mathrm{c}$ ). This switching method reduce the delay to $2,3344 \mathrm{~ms}$ until reach $0,4608 \mathrm{~ms}$.

\section{CONCLUSION}

This paper presents recent results on NCS. This new domain of research gathers different scientific communities such as control and communication specialists. The goal of this paper is to propose a new integrated modelling environment for NCS. A method based on the adaptation of the weights of WRR algorithms implemented in the Ethernet switch is developed. This modelling makes use of the high Petri nets formalism. A NCS was modelled by the HTCPN based on an extension of Ethernet switch model. The controller, actuator, process and sensor are modelled and integrated to the Ethernet switch model. A strategy to control the network by adapting the quality of service according to the quality of control required by the application is proposed. This strategy is implemented on the basis of the WRR mechanism. To control the network, a comparison of frames delay to a 
threshold delay tolerated by the feedback system is fulfilled. Then, the weights of the WRR implemented in the Ethernet switch are changed to reduce the network induced delay. The simulation results show that the control of network, improves the overall system performances. The modular and generic aspects of the HTCPN model enable to integrate other protocols, controllers, processes... The perspective of this work is to use our model for proposing more sophisticated algorithms for adjusting QoS and QoC by controlling in coordinated way both the network and the process.

a)
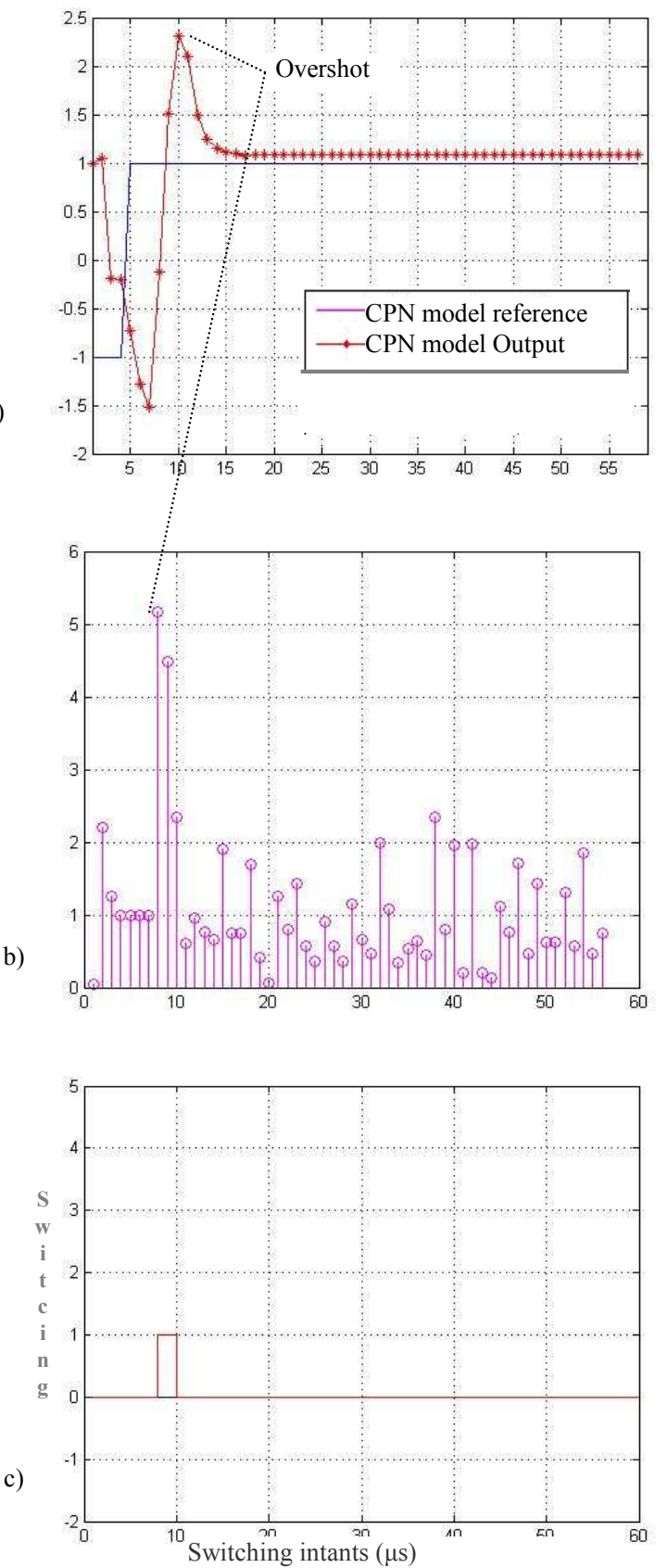

Fig. 7. Output a), delay ( $\mu \mathrm{s})(\mathrm{b})$ and switching instants (c) of HTCPN model and Matlab model, at 0.7 environment load.

\section{ACKNOWLEDGMENT}

This research has been conducted as a part of the Networked Control Systems Tolerant to faults (NeCST) Project contract $\mathrm{n}^{\circ}$ IST - 2004-004303.

\section{REFERENCES}

[1] M.S. Branicky, S. M. Phillips and W. Zhang, Stability of networked Control systems: Explicit Analysis of Delay, In Proc. of the American Control Conference (ACC'2000), Chicago, Illinois, June 2000.

[2] B.Brahimi, C.Aubrun and E.Rondeau (2006). Modelling of scheduling policies implemented in Ethernet switch by using Coloured Petri Nets. In proc. of 11th IEEE International Conference on Emerging Technologies and Factory Automation Prague, Czech Republic, 2006.

[3] E. Altman, T. Bas,ar, and R. Srikant, "Congestion control as a stochastic control problem with action delays," Automatica, vol. 35, pp. 1937-1950, Dec. 1999.

[4] S. Mascolo, "Congestion control in high -speed communication networks using the smith predictor principle," in Automatica, vol. 35, $\mathrm{n}^{\circ} 12$, dec. 1999.

[5] F. Göktas, Distributed control systems over a communication networks, PhD. Dissertation, université de Pennsilvanie, 2000.

[6] J. Filipiak, Modelling and control of dynamic flows in communication networks, Berlin: Springer, 1988.

[7] J-P. Georges, T. Divoux et E. Rondeau, Evaluation d majorants des délais de transmission pour les systèmes contrôlés en réseau, Traité IC2 Information-Commande-Communication, Hermès Science, Lavoisier, $\mathrm{N}^{\circ}$ ISBN 978-2-7462-1513-9, 2007, Chapitre 5, pp.189230.

[8] G.C. Walsh, H. Ye and L. Bushnell, Stability Analysis of Networked Control Systems, In Proc. American Control Conference, Chicago, pp. 2876-2880, June 1999.

[9] A. Koubaâ, Gestion de la Qualité de Service temporelle selon la contrainte $(\mathrm{m}, \mathrm{k})$-firm dans les réseaux à commutation de paquets, LORIA-TRIO, thèse octobre 2004.

[10] H. Hoang, M. Jonsson, A. Larsson, R. Olsson, et C. Bergenhem. Deadline First Scheduling in Switched Real-Time Ethernet- Deadline Partitionning Issues and Software Implementation Experiments. In proc. Of $1^{\text {st }}$ Intl. Workshop on Real-Time LANs in the Internet Age Satellite Event to $14^{\text {th }}$ Euromicro Conference on Real- Time Systems Technical University of Vienna, Austria, 18 juin 2002.

[11] S-K. Kweon, K-G. Shin and Q. Zheng, Statistical Real-time communication over Ethernet for manufacturing automation systems, in proc. Of IEEE real-time Technology and Applications Symposium (RTAS'1999), pp. 192-202, Juin 1999.

[12] Lo Bello, L., Mirabella, O.,., "Fuzzy Traffic Smoothing: an Approach for Real-time Communication over Ethernet Networks", Proc of WFCS 2002，4 $4^{\text {th }}$ IEEE Workshop on Factory Communication Systems", Västeras, Sweden, August 2002.

[13] M. E.M. Ben Gaid, A. Çela et Y. Hamam, Optimal Integrated Control and Scheduling of Networked Control Systems with Communication Constraints: Application to a Car Suspension System, In pro. Of IEEE Transactions On Control Systems Technology, Vol. $14 \mathrm{~N}^{\circ}$ 4, July 2006.

[14] M. S. Branicky, S. M. Phillips and W. Zhang, Scheduling and feedback co-design for networked control systems, in Proc. of the IEEE Conference on Decision and Control, December 2002.

[15] G. Marsal, Evaluation of time performances of Ethernet-based Automation System by simulation of High Level Petri Nets, PhD dissertation de l'Ecole Normale Supérieure de Cachan et de l'Universté de Kaiserslautern, Décembre 2006.

[16] S. Abdellatif and G. Juanole, Evaluation of quality of service of Periodic Producer/Consumer Relationship implemented on an ATM LAN, In Emerging Technologies and Factory Automation, 1999. Proceedings. ETFA '99. 1999.

[17] B. Brahimi, Proposition d'une approche intégrée basée sur les réseau de Petri de haut niveau pour simuler et evaluer les systèmes contrôlés en réseau, PHD dissertation at CRAN, December 2007.

[18] Nilsson, Real Time Control Systems with Delays, Ph.D. dissertation, Dept. Automatic Control, Lund Institut of Technology, Lund, Sweden, January 1998.

[19] B. Wittenmark, J. Nilsson and M. Törngren, Timing problems in realtime control systems, American Control Conference, Seattle, Washington, 1995. 